\title{
DESARROLLO DE LECCIONES SOBRE REACTORES QUÍMICOS Y BIOLÓGICOS EN LA PLATAFORMA MOODLE
}

\section{DEVELOPMENT OF LESSONS ON CHEMICAL AND BIOLOGICAL REACTORS IN MOODLE PlatForm}

\author{
Inés María Santos-Dueñas, Isidoro García-García, \\ Juan Carlos García-Mauricio, Alejandro Rodríguez-Pascual, \\ Luis Jiménez-Alcaide, José Ramos-Ruiz, Juan José Moreno-Vigara, \\ Ana María Cañete-Rodríguez, Eduardo Espinosa-Víctor, \\ Juan Domínguez-Robles, Rafael Sánchez-Serrano, \\ Zoilo González-Granados, Gema María Varo-Sánchez, \\ Laura Ramos Moreno, Jaime Moreno García, \\ María Teresa García-Martínez \\ ines.santos@uco.es \\ Universidad de Córdoba
}

Received: 30/06/2017Ａccepted: 04/12/2017

\begin{abstract}
In this teaching project have developed a series of academic activities devoted to the chemical and biological reactors, in particular, it has been using a tool called lesson which is available through the Moodle virtual platform, these lessons are based on a series of sequential windows allowing to delve into all the fundamental concepts of chemical and/or biological reactors and through various complementary on the theme, including issues during the development of this lesson, students perform a more active learning that promotes the consolidation of concepts gradually.

Prior to the study of chemical reactors and/or biological is necessary to take account of various chemical, physical, mathematical, biological and engineering aspects that are to be considered to develop the basic fundamentals to achieve the aim and is intended to facilitate this work to the students with the use of elaborate lessons.

Important preparation work has been prior to the preparation of the lessons and synthesis of information, have subsequently developed different lessons for the concepts dealt with in each of the issues involved in the development of this educational project and after a thorough review have been available to the students.
\end{abstract}

Keywords: Chemical reactors; Bioreactors; Moodle; Lesson.

\section{Resumen}

En este proyecto docente se han desarrollado una serie de actividades académicamente dirigidas dedicadas a los reactores químicos y biológicos, 
en concreto, se ha empleado una herramienta denominada lección que se encuentra disponible a través de la plataforma virtual Moodle, dichas lecciones se basan en una serie de ventanas secuenciales que permiten profundizar en todos los conceptos fundamentales de los reactores químicos y/o biológicos $\mathrm{y}$, a través de diversas cuestiones complementarias sobre el tema, incluidas durante el desarrollo de dicha lección, permite al alumno realizar un aprendizaje más activo que favorece la consolidación de los conceptos de forma gradual.

Previo al estudio de los reactores químicos y/o biológicos es necesario tener en cuenta diversos aspectos químicos, físicos, matemáticos, biológicos e ingenieriles que han de considerarse para desarrollar los aspectos fundamentales básicos para poder alcanzar dicho objetivo y se pretende facilitar esta labor al alumnado con el empleo de las lecciones elaboradas.

Previamente a la elaboración de las lecciones se ha realizado una importante labor de preparación y síntesis de toda la información, posteriormente se han desarrollado diferentes lecciones para los conceptos tratados en cada uno de los temas implicados en la elaboración de este proyecto docente y tras una profunda revisión se han puesto a disposición de los alumnos. Lección.

Palabras clave: Reactores químicos; Reactores biológicos; Moodle;

\section{INTRODUCCIÓN}

Entre algunos de los aspectos más importantes que promueve el Espacio Europeo de Educación Superior (EEES) se destaca la promoción de metodologías de enseñanza-aprendizaje que se ajusten a las nuevas realidades y que incentiven una participación más activa y corresponsable por parte del alumnado. Entre estas y otras razones, el profesorado implicado en este proyecto ha tratado de introducir en sus clases nuevos modelos de enseñanzaaprendizaje que potencien la actividad, implicación y autonomía del alumnado. Para ello, se han incorporado estrategias de enseñanza que aportan una manera diferente de entender la enseñanza $y$, con ello, la universidad y sus relaciones con la sociedad según queda recogido en La Ley Orgánica 4/2007, de 12 de abril, por la que se modifica la Ley Orgánica 6/2001, de 21 de diciembre, de Universidades (LOMLOU).

En este contexto se ha considerado muy importante que el alumnado sea capaz de profundizar en uno de los aspectos claves de muchos procesos industriales y/o biotecnológicos como es el conocimiento de los reactores químicos y/o biológicos. En este proyecto se han desarrollado unas actividades académicamente dirigidas empleando una herramienta disponible a través de la 
plataforma virtual Moodle denominada "Lección" que permite al alumno, a través de varias ventanas secuenciales, profundizar en todos los conceptos fundamentales $\mathrm{y}$, a través de diversas cuestiones sobre el tema, verificar que se han comprendido las ideas desarrolladas.

\subsection{PRESENTACIÓN DE LAS ASIGNATURAS IMPLICADAS}

El estudio de los aspectos fundamentales de los reactores químicos y/o biológicos implica tener un conocimiento previo sobre diversos aspectos químicos, físicos, matemáticos, biológicos e ingenieriles que sugieren la necesidad de implicar a un amplio equipo docente que permita enriquecer el proyecto desarrollado, además, gracias a la participación docente en varias asignaturas de diversas titulaciones de la Universidad de Córdoba en las que, en mayor o menor grado, los reactores químicos y/o biológicos son una parte importante de los temarios ha permitido poder llegar a un mayor número de alumnos.

Las asignaturas (por orden alfabético) con temas dedicados a reactores químicos y/o biológicos que han sido objeto de las lecciones elaboradas en este proyecto docente son las siguientes:

- Bioquímica y Microbiología Industriales de tercer curso del Grado de Bioquímica (Obligatoria).

- Tema 9. Introducción a los reactores biológicos

- Tipos de fermentadores. Diseño de un fermentador.

- Fundamentos de Ingeniería Química de segundo curso del Grado de Ciencia y Tecnología de los alimentos (Obligatoria)

- Bloque 4. Reactores y Biorreactores. Una Introducción.

- Introducción. Clasificación de las Reacciones Químicas. Termodinámica. Cinética. Tipos de reactores ideales. Reactor Discontinuo de Mezcla Completa (RDMC). Reactor Continuo de Mezcla Completa (RCMC). Reactor Continuo de Flujo Pistón (RCFP).

- Ingeniería Química II de cuarto curso del Grado de Química (Obligatoria).

- Tema 5. Introducción. Conceptos generales. Balance de materia con reacción química. Velocidad de reacción. Determinación de la ecuación cinética.

- Tema 6. Diseño de reactores ideales. Reactor discontinuo. Reactor continuo de mezcla completa. Reactor de flujo pistón.

- Tema 7. Reactores para reacciones simples. Selección del reactor más adecuado. Asociación de reactores. Reactores para reacciones múltiples. 
- Tema 8. Reactores no isotérmicos. Balances de materia y energía: reactor discontinuo, continuo de mezcla perfecta y continuo de flujo pistón.

- Microbiología y Biotecnología Industrial de tercer curso del Grado de Ingeniería Agroalimentaria y del Medio Rural (Obligatoria).

- Tema 6. Biorreactores. Procesos continuos y discontinuos

- Reactores Biológicos de cuarto curso del Grado de Ciencia y Tecnología de los Alimentos (Optativa).

- Tema 1. Biorreactores e industrias alimentarias.

- Tema 2. Tipos de biorreactores.

En este trabajo se pretende presentar una propuesta de mejora del proceso de enseñanza-aprendizaje mediante el empleo de lecciones, a través de la plataforma virtual Moodle de cada asignatura, para consolidar los conceptos teóricos tratados en Gran Grupo antes de realizar los diferentes seminarios prácticos programados en cada caso.

\section{OBJETIVOS}

Por parte del alumnado durante el desarrollo de este proyecto se ha trabajado en los siguientes objetivos:

- Adquirir conocimientos y destrezas para el manejo de nuevas tecnologías.

- Profundizar en los aspectos fundamentales sobre los reactores químicos y/o biológicos.

- Incentivar los procesos de autoaprendizaje y autoevaluación por parte del alumnado.

- Promover un aprendizaje activo y gradual por parte de los alumnos.

- Mejorar los resultados académicos obtenidos por los alumnos en las asignaturas implicadas.

Por otro lado, el personal docente que ha formado parte de este proyecto docente ha profundizado, además, estos otros objetivos:

- Desarrollar técnicas de enseñanzas innovadoras englobadas dentro del modelo de EEES en la Universidad de Córdoba.

- Mejorar los procesos de enseñanza-aprendizaje llevados a cabo por los alumnos.

- Incorporar el empleo de las nuevas tecnologías en los procesos de enseñanza-aprendizaje.

- Promover el empleo de diversas herramientas de la plataforma virtual Moodle.

- Interaccionar con equipos docentes multidisciplinares. 
- Elaborar material digital sobre conceptos fundamentales en el estudio de los reactores químicos y/o biológicos.

\section{METODOLOGÍA}

La metodología empleada en este proyecto tiene dos etapas claramente diferenciadas:

- Una primera, llevada a cabo por el profesorado, de preparación de la información para la elaboración de las lecciones, $y$

- Una segunda, llevada a cabo por el alumnado, de puesta en práctica las lecciones elaboradas en las diferentes plataformas virtuales Moodle disponibles para cada asignatura.

El proyecto ha implicado el desarrollo de una serie de actividades empleando mayoritariamente el uso de las nuevas tecnologías que se detallan a continuación clasificadas dentro de su etapa correspondiente.

\subsection{PREPARACIÓN DE LA INFORMACIÓN}

1. Preparación y síntesis de la información sobre reactores químicos y/o biológicos.

a. Tipos de reacción y estado físico de reactivos a la entrada

i. Homogéneas, heterogéneas, catalizadas, no catalizadas...

b. Tipo de reactor

i. Homogéneo, heterogéneo, discontinuo, continuos, semicontinuos, isotérmicos, adiabáticos...

c. Aspectos físico-químicos fundamentales de la reacción

i. Equilibrio químico y/o bioquímico, cinética química y/o bioquímica...

2. Esquematización de la información en diferentes bloques secuenciales.

3. Preparación de cuestiones para comprobar el conocimiento adquirido por el alumnado.

4. Elaboración de las lecciones con cuestiones incluidas a través de la herramienta. 
5. Revisión de las lecciones elaboradas por todos los componentes del proyecto docente, tanto desde un punto de vista operativo como conceptual.

6. Correcciones de las lecciones elaboradas con los comentarios de todos los componentes del proyecto docente.

\subsection{PUESTA EN PRÁCTICA DE LAS LECCIONES ELABORADAS}

1. Inserción de las lecciones en el aula virtual Moodle de las distintas asignaturas implicadas y realización por parte del alumnado.

2. Realización de una encuesta a los alumnos sobre los posibles beneficios del empleo de las lecciones elaboradas.

3. Realización de una acción tutorial para contribuir a la resolución de las dudas y dificultades que los estudiantes pudieran encontrar a la hora de manejar las diferentes lecciones elaboradas.

\section{DESCRIPCIÓN}

Las diferentes fases de elaboración de las distintas lecciones han sido similares entre sí, diferenciando entre cada una de ellas el tema en concreto y, por lo tanto, el contenido de las diferentes ventanas secuenciales y, consecuentemente, la lección final creada.

\subsection{PREPARACIÓN Y SÍNTESIS DE LA INFORMACIÓN SOBRE REACTORES QUÍMICOS Y/O BIOLÓGICOS}

En esta primera fase se ha realizado una profunda labor de síntesis y de estructuración de la materia que debía de contener cada una de las lecciones y ha sido el material que se ha empleado como base en las siguientes fases.

\subsection{ESQUEMATIZACIÓN DE LA INFORMACIÓN EN DIFERENTES BLOQUES SECUENCIALES}

Como el desarrollo de las lecciones se lleva a cabo mediante diferentes ventanas secuenciales se ha realizado un esquema del contenido que se quería recoger en cada una de las lecciones, además, en algunos casos se han utilizado diferentes rutas de desplazamiento dentro de las propias lecciones en función de las necesidades del alumnado en cada momento. 
4.3.PREPARACIÓN DE CUESTIONES PARA COMPROBAR EL CONOCIMIENTO ADQUIRIDO POR EL ALUMNADO

De manera complementaria al contenido teórico de las diferentes lecciones se han elaborado varias cuestiones para que los alumnos pudieran ir comprobando el grado de asimilación de los diferentes conceptos tratados y, consecuentemente, poder guiar al alumno por una u otra secuencia de ventanas dentro de la propia lección según las necesidades concretas en cada caso.

\subsection{ELABORACIÓN DE LAS LECCIONES CON CUESTIONES INCLUIDAS A TRAVÉS DE LA HERRAMIENTA}

Una vez que se ha preparado toda la documentación por el profesorado participante en este trabajo en las tres etapas previas se ha ido implementando en la plataforma Moodle de cada asignatura las diferentes lecciones. La utilidad de cada una de las diferentes lecciones creadas se basa en la gran labor de planificación, estructuración y síntesis de las etapas anteriores.

4.5.REVISIÓN DE LAS LECCIONES ELABORADAS POR TODOS LOS COMPONENTES DEL PROYECTO DOCENTE, TANTO DESDE UN PUNTO DE VISTA OPERATIVO COMO CONCEPTUAL

Esta etapa es fundamental para comprobar el correcto funcionamiento de las lecciones, se han ido comprobando las diferentes alternativas posibles en cada una de las distintas lecciones y se han recopilado todas las incidencias observadas.

4.6.CORRECCIONES DE LAS LECCIONES ELABORADAS CON LOS COMENTARIOS DE TODOS LOS COMPONENTES DEL PROYECTO DOCENTE

Se han corregido todas las incidencias observadas en la etapa anterior, tanto desde el punto de vista de avance entre las distintas ventanas dentro de la lección como algunas erratas del material elaborado al subirlo a la plataforma Moodle. También, se han reestructurado algunas ventanas para facilitar el aprendizaje secuencial de los alumnos.

La labor de todos los miembros del equipo del proyecto en las etapas de revisión y corrección han sido fundamentales para obtener unas lecciones que realmente sean útiles para el alumnado. 
4.7.INSERCIÓN DE LAS LECCIONES EN EL AULA VIRTUAL MOODLE DE LAS DISTINTAS ASIGNATURAS IMPLICADAS Y REALIZACIÓN POR PARTE DEL ALUMNADO

En esta etapa se ha logrado tener una lección definitiva para cada uno de los temas y cada una de ellas se ha puesto en modo visible para permitir el acceso a los alumnos cuando era adecuado para el desarrollo de cada asignatura.

4.8.REALIZACIÓN DE UNA ENCUESTA A LOS ALUMNOS SOBRE LOS POSIBLES BENEFICIOS DEL EMPLEO DE LAS LECCIONES ELABORADAS

Una vez se comprueba que la lección ha sido realizada por un número adecuado de alumnos en cada uno de los diferentes temas se han realizado diferentes encuestas a través de una herramienta de Moodle denominada "Consulta" que permite de forma anónima responder a una serie de preguntas para obtener una opinión real del alumnado en aspectos que el profesorado ha considerado importantes, tales como grado de utilizad de la lección, facilidad de manejo por el alumnado, empleo de la herramienta para consolidar los conceptos antes de pasar a los diferentes contenidos prácticos de cada tema, entre otras.

\subsection{REALIZACIÓN DE UNA ACCIÓN TUTORIAL PARA CONTRIBUIR A} LA RESOLUCIÓN DE LAS DUDAS Y DIFICULTADES QUE LOS ESTUDIANTES PUDIERAN ENCONTRAR A LA HORA DE MANEJAR LAS DIFERENTES LECCIONES ELABORADAS

Paralelamente a la puesta a disposición de los alumnos de las diferentes lecciones se ha desarrollado una acción tutorial para poder ayudar al alumnado en la utilización de las diferentes lecciones, no solo para poder afianzar los conocimientos adquiridos en las clases de Gran Grupo sino también para repasarlos a lo largo del desarrollo de la asignatura y previos a la realización del examen oficial.

\section{RESULTADOS OBTENIDOS}

Se han creado nueve lecciones en cinco asignaturas diferentes de cuatro titulaciones oficiales de Grado de la Universidad de Córdoba. A través del empleo de dichas lecciones por el alumnado se ha mejorado el conocimiento sobre los reactores químicos y biológicos, y gracias a las cuestiones de las diferentes lecciones se ha conseguido una autoevaluación por parte los alumnos que ha 
permitido poder aclarar algunos conceptos claves durante el desarrollo de la docencia.

El empleo de herramientas distintas a las habituales en la mayoría de las asignaturas (lecciones, consultas...), e incluso en los diferentes cursos académicos, favorece el grado de motivación de los alumnos en estas asignaturas pues dichos alumnos valoran muy positivamente un mayor grado de implicación del profesorado por innovar en la labor docente cotidiana.

Y este mayor grado de motivación por los alumnos favorece una mayor participación de los mismos no solo en las diferentes actividades propuestas en la plataforma virtual Moodle sino hacen que tengan una actitud más activa en las clases favoreciendo unas clases más dinámicas y participativas.

En los anexos de la memoria se muestra un breve resumen del tipo de material elaborado, pero al elaborarse un total de nueve lecciones es muy extenso y, además, al ser totalmente virtual a través de la plataforma Moodle no puede detallarse por completo.

\section{UTILIDAD/ANÁLISIS}

El desarrollo de las diferentes actividades de este proyecto docente ha facilitado una renovación en el material de diversos temas de Reactores químicos y biológicos.

\subsection{UTILIDAD PARA EL PROFESORADO}

Las diferentes actividades de sintesis, estructuración y esquematización de la información en las diversas etapas llevadas a cabo en este trabajo ha permitido una labor de trabajo conjunta que ha enriquecido mucho el grupo de trabajo desde un punto de vista docente.

Ha favorecido una renovación del material docente en la plataforma Moodle al incluir las diferentes lecciones y encuestas, y se ha visto que existe un gran potencial en la plataforma Moodle que no se explota por el profesorado.

\subsection{UTILIDAD PARA EL ALUMANDO}

La utilización de las diferentes lecciones por el alumnado ha facilitado la adquisición gradual de conocimientos fundamentales previos al desarrollo de diferentes sesiones prácticas de las distintas asignaturas. 
El empleo de herramientas distintas y las cuestiones surgidas durante el mismo han favorecido un aprendizaje más dinámico por el alumnado.

La labor tutorial llevada a cabo durante el desarrollo de las lecciones ha permitido un acercamiento con el alumno que ha favorecido un mayor grado de implicación de la asignatura.

\section{CONCLUSIONES/DISCUSIÓN}

La base fundamental de este proyecto docente se basa en el empleo de las nuevas tecnologías, en concreto en una aplicación de la plataforma virtual Moodle que es la utilizada por la Universidad de Córdoba para sus Estudios Oficiales de Grado y Postgrado. El empleo de dicha plataforma ha sido llevado a cabo tanto por el profesorado implicado como para el alumnado de todas las asignaturas implicadas.

Este proyecto ha permitido la colaboración entre un amplio equipo docente que ha enriquecido mucho el material elaborado, además, al tener una gran variedad de alumnos que han podido trabajar con el material desarrollado se han analizado las ventajas y desventajas del empleo de las lecciones.

Si bien, es una herramienta que necesita una gran cantidad de trabajo para su planificación, estructuración y elaboración por parte del profesorado, por otro lado, permite al alumno favorecer su proceso de autoaprendizaje, asi como, afianzar los conocimientos adquiridos en clase, y, además, aumenta el grado de motivación de los alumnos.

Adicionalmente, al incluir varias cuestiones en distintas fases durante el desarrollo de la lección se permite una autoevaluación de los diferentes conceptos implicados en cada una de las lecciones por parte de los alumnos y según las diferentes respuestas se dirige al alumno mediante distintos itinerarios de aprendizaje para que pueda repasar los conceptos necesarios para mejorar en dicha autoevaluación.

Los alumnos de los diferentes grados, especialmente en los últimos cursos, están muy saturados de actividades de las diferentes asignaturas y la ventaja del empleo de las lecciones como actividad virtual no aumenta la carga de trabajo por el alumno sino que facilita y favorece el estudio de los conceptos de los diferentes temas.

Mediante la implementación de las diferentes lecciones propuestas durante el curso académico se ha observado una mejora en los resultados académicos globales de los alumnos. 
Para el profesorado ha sido una experiencia muy enriquecedora desde un punto de vista pedagógico y de profundización en los diferentes contenidos, si bien, sería interesante aplicar también la herramienta lección en la preparación de seminarios, prácticas de laboratorio, resolución de problemas... para reforzar el aprendizaje del alumnado en las diferentes actividades llevadas a cabo para la adquisición de competencias en cada una de las distintas asignaturas.

\section{AgRADECIMIENTOS}

Este trabajo ha sido financiado gracias al Proyecto de Innovación Docente 2016-1-2007 concedido en la Modalidad 1 del Plan de Innovación y Buenas Prácticas Docentes. Curso 2016/2017 por la Universidad de Córdoba.

\section{BIBLIOGRAFÍA}

Brown, CM. CAMPBeLL, I. PRIEST, FG. (última ed.). Introducción a la Biotecnología. Ed. Acribia, Zaragoza.

Calleja, G., García, F., Lucas, A., Prats, D., RodríGuez, JM. Introducción a la Ingeniería Química. Ed. Síntesis. 1999.

DenBigh, KG. TURNER, JCR. Introducción a la teoría de los reactores quimicos. Ed. Limusa. México. 1990.

FOGLER, HS. Elementos de Ingeniería de las Reacciones Quimicas. Prentice Hall. $3^{\mathrm{a}}$ ed. 2001.

LEE, BH. Fundamentos de la Biotecnología de los Alimentos. Ed. Acribia, Zaragoza, 2000.

LeVEAU, JY. BOUIX, M. Microbiología Industrial. Ed. Acribia, Zaragoza, 2000.

LEVESPIEL, O. Ingeniería de las reacciones químicas. Ed. Reverté S.A. Barcelona, 1990.

OKAFOR, N. Modern Industrial and Biotechnology. CRC Press Taylor \& Francis Group, New York, 2007.

SALMI, TO., MikKOLA, JP, WÄRNA, JP. Chemical Reaction Engineering and Reactor Technology. CRC Press, 2011

Sмith, JM. Chemical engineering kinetics. McGraw-Hill. Tokyo. 1982. 
ANEXOS:

Anexo I. Capturas de la ventana de edición de una página de contenidos de una lección.

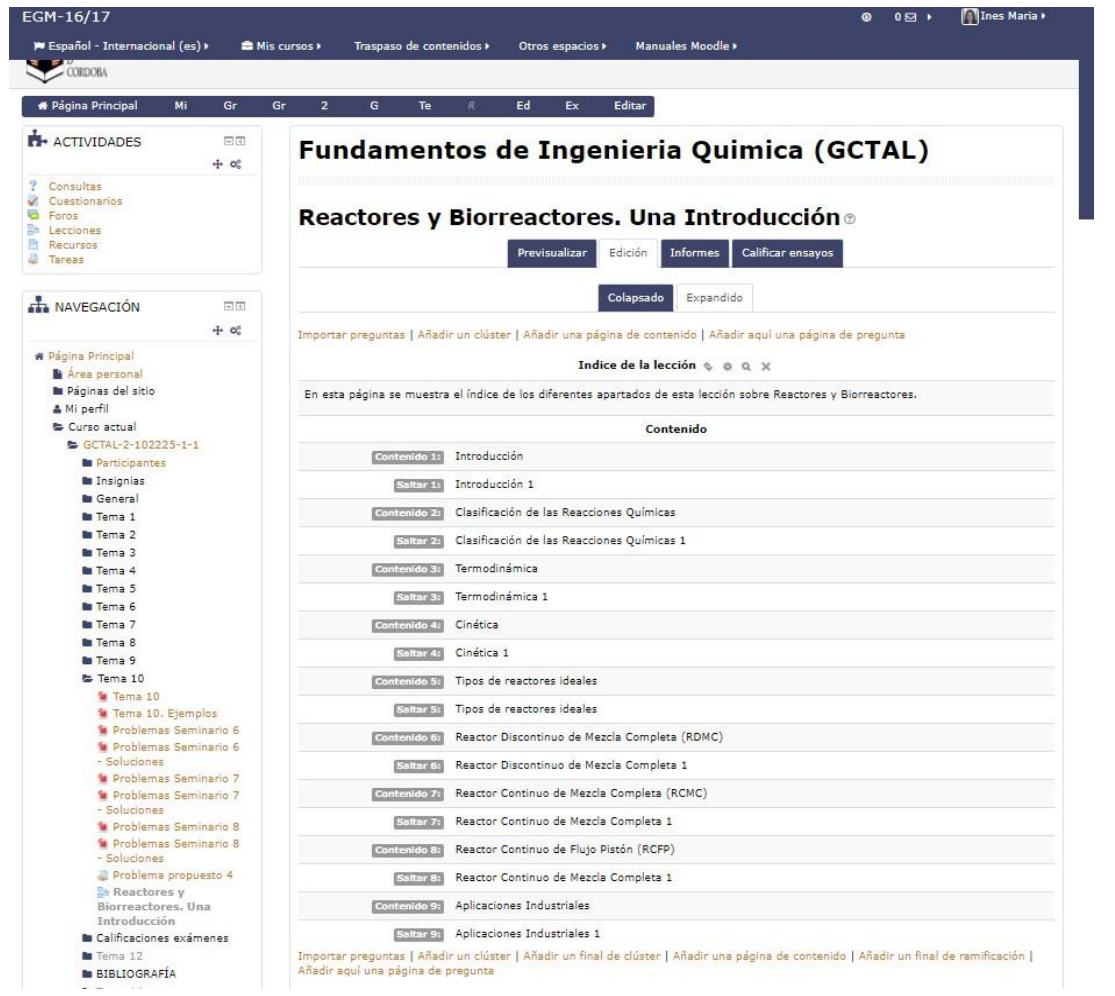

AI.1. Captura de pantalla de la Vista de edición de una página de contenido. 


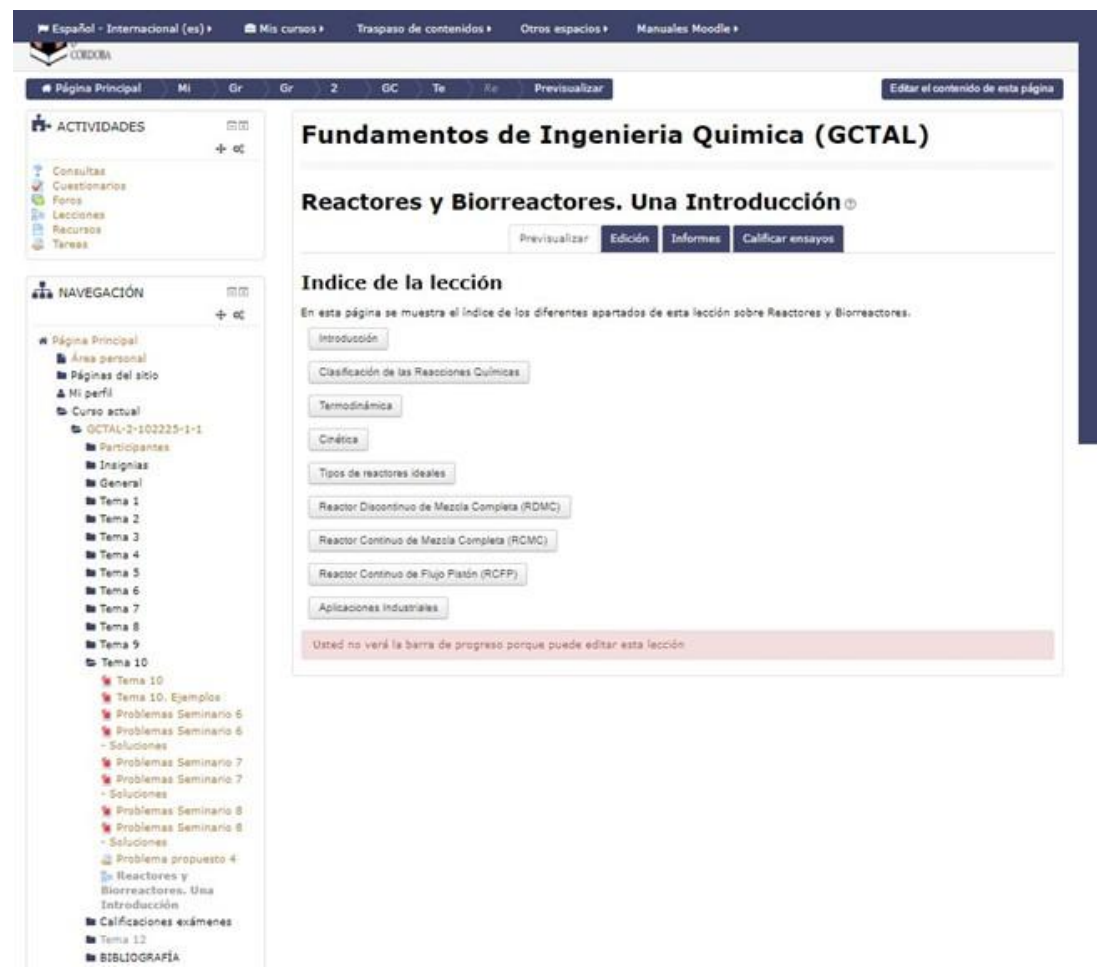

AI.2. Captura de pantalla de la Vista de previsualización de una página de contenido.

Anexo II. Capturas de la ventana de edición de una pregunta de verdadero/falso de una lección.

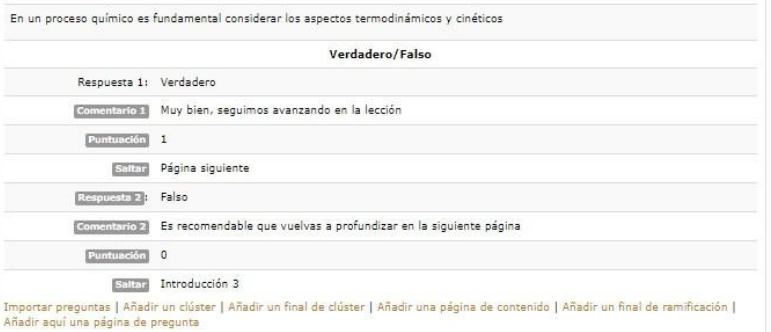

Importar preguntas | Añadir un clústar | Añadir un final de dúster | Añadir una página de contenido | Añadir un final de ramificación |

AII.1. Captura de pantalla de la Vista de edición de una página de pregunta. 


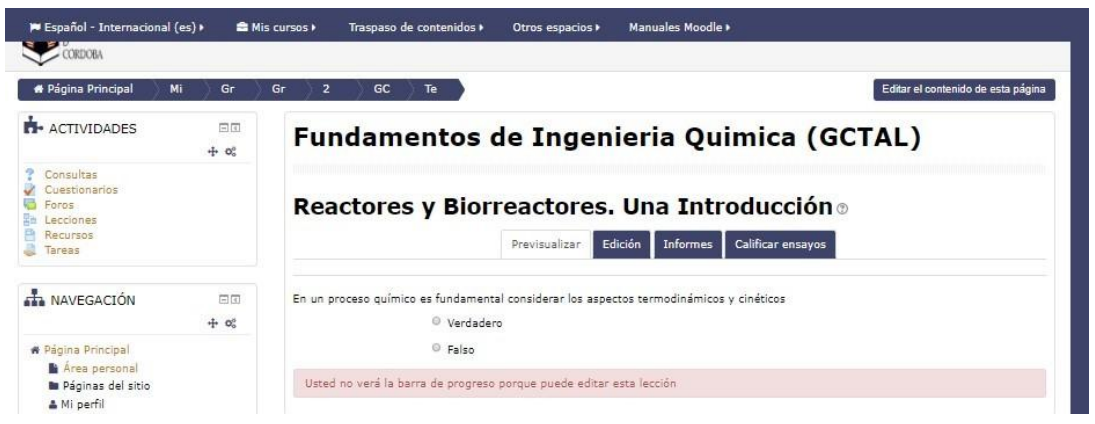

AII.2. Captura de pantalla de la Vista de previsualización de una página de pregunta.

Anexo III. Consulta tipo.

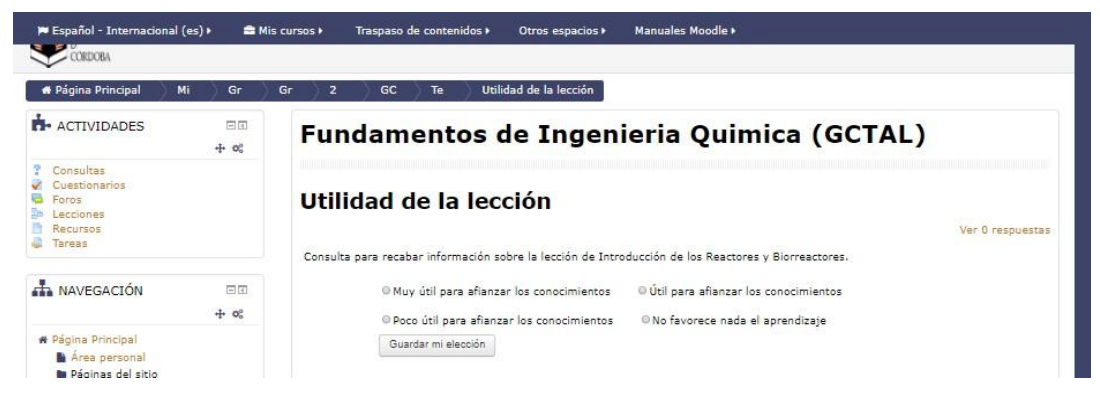

AIII.1. Captura de pantalla de una consulta sobre la utilidad de la lección. 